\title{
CELL MigRATION
}

\section{Regulating the (missing) link}

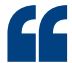

... ACF7, under the dynamic control of

GSK3 $\beta$, can regulate

sustained

directional

microtubule

growth, cell

polarity and

migration ...
Wu et al. report in Cell that glycogen synthase kinase $3 \beta(\mathrm{GSK} 3 \beta)$ regulates an interaction between actincrosslinking factor 7 (ACF7; also known as MACF1) and microtubules, further bolstering its status as a key mediator of cell polarity and cytoskeletal dynamics. They outline this mode of regulation using as a model the migration of stem cells (SCs) from a specific niche of the hair follicle, the bulge, in response to injury.

The authors observed an electrostatic interaction between the acidic carboxy-terminal tails of tubulin subunits and the basic C-terminal tail of ACF7, which contains consensus phosphorylation sites for GSK3 $\beta$. Constitutive GSK $3 \beta$ activation or the presence of a phosphomimetic version of ACF7 decreased the affinity of ACF7 for microtubules, whereas

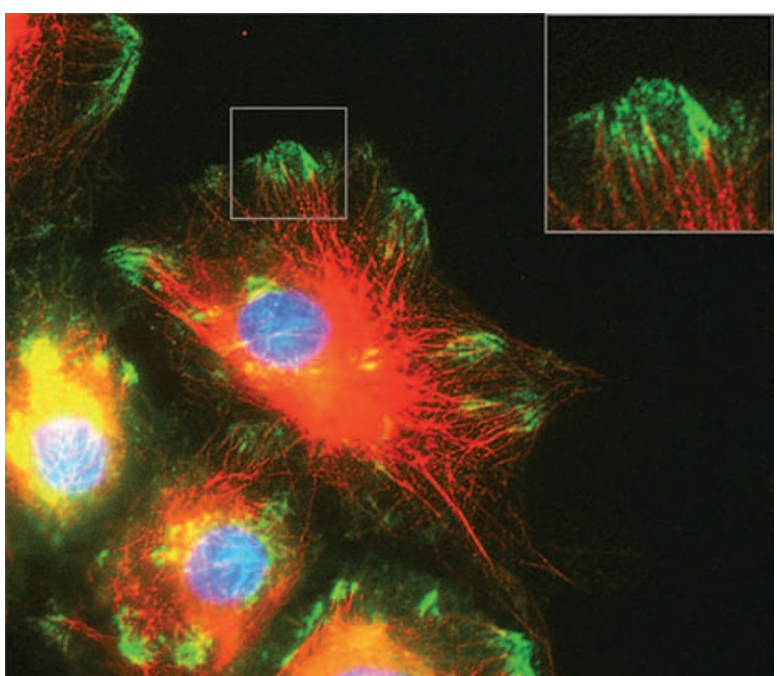

Immunofluorescence staining of wild-type hair follicle bulge stem cells treated with the glycogen synthase kinase $3 \beta$ inhibitor lithium chloride. Note the enhanced localization of actin-crosslinking factor 7 (also known as MACF1; green) at the plus ends of microtubules (red). Nuclei were counterstained with 4,6-diamidino-2 -phenylindole (DAPI; blue). Image courtesy of X. Wu, Rockefeller University, New York, USA.
GSK3 $\beta$ inhibition or the use of a kinase-refractile version of ACF7 retained microtubule-binding affinity. This also happened in cultured keratinocytes, in which constitutive GSK $3 \beta$ activation reduced the localization of ACF7 along microtubules and resulted in an irregular microtubule network. Conversely, on GSK3 $\beta$ inhibition, ACF7 concentrated at microtubule ends at the cell periphery.

WNT signalling inhibits GSK3 $\beta$ and has also been implicated in wound repair, so Wu et al. tested whether this process might be linked to ACF7 regulation. Loss of ACF7 specifically in bulge SCs (by conditional knockout) or inhibition of GSK3 $\beta$ on wounded wild-type skin perturbed epidermal wound healing in mice.

The authors had previously shown that cultured keratinocytes lacking ACF7 could not coordinate microtubule growth along actin filaments, so they hypothesized that regulated ACF7-microtubule interactions might be needed for cytoskeletal modelling and consequent polarized migration. In a modified Boyden chamber assay, hair follicle SCs lacking ACF7 showed impaired directional movement. Exploring how ACF7-microtubule interactions contribute to cell polarity, the team showed that although polarization of phosphorylated (inactivated) GSK3 $\beta$ was seen at the leading edge of both wild-type and ACF7-deficient hair follicle SCs, only the wild-type cells polarized perinuclear Golgi. This placed ACF7 downstream of GSK3 $\beta$ inhibition at the migrating edge but upstream of features of cellular polarization. Moreover, only wildtype ACF7 restored both polarization and migration in ACF7-deficient hair follicle SCs - neither the phosphomimetic version nor the kinase-refractile version alone or in combination rescued the phenotype. This implies a need for the dynamic regulation of ACF7 phosphorylation.

Turning again to physiological relevance, Wu et al. similarly found that, in mice, only wild-type ACF7 could restore directional hair follicle SC movement from the bulge to facilitate wound repair. Furthermore, in skin tissue explants, ACF7-deficient cells were inefficient at polarizing and migrating out of the bulge, and microtubule growth was randomized. By contrast, wild-type bulge SCs had well-polarized microtubule bundles that co-aligned with filamentous actin, and movements were polarized. Notably, phosphorylated GSK3 $\beta$ was enriched at the leading edge of these cells.

So, Wu et al. have identified that the spectraplakin ACF7, under the dynamic control of GSK $3 \beta$, can regulate sustained directional microtubule growth, cell polarity and migration in skin SCs. This capacity enables them to efficiently rebuild the epithelium in response to wounding, a process in which WNT signalling is elevated and GSK3 $\beta$ is locally inhibited.

Katrin Legg, Gateway Assistant Editor, Cell Migration Gateway

ORIGINAL RESEARCH PAPER Wu, X. et al. Skin stem cells orchestrate directional migration by regulating microtubule-ACF7 connections through GSK3ß. Cell 144, 341-352 (2011) 